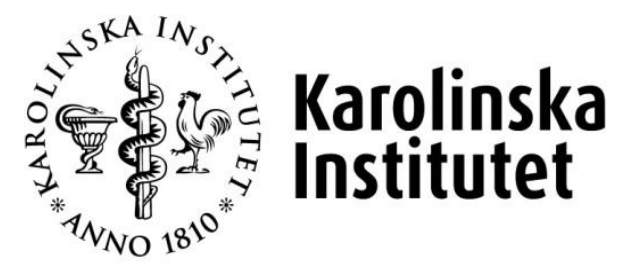

Institutionen för klinisk neurovetenskap

Psykologprogrammet, termin 6

Huvudämne: Psykologi

Examensarbete (C-nivå) i psykologi (2PS013), 15 poäng

Vårterminen 2017

\title{
Ethical review board-approved protocols and intent to use open practices in research on human subjects in Sweden
}

Arian Jafari \& Bo Jenner

Handledare: Gustav Nilsonne, Karolinska Institutet, Institutionen för klinisk neurovetenskap Bihandledare: Rebecca Willén, IGDORE, The Globally Distributed Institute for Open Research and Education Examinator: Johan Lundström, Karolinska Institutet, Institutionen för klinisk neurovetenskap 


\section{Institutionen för klinisk neurovetenskap}

Psykologprogrammet, termin 6

Huvudämne: Psykologi

Examensarbete (C-nivå) i psykologi (2PS013), 15 poäng

Vårterminen 2017

\section{Ethical review board-approved protocols and intent to use open practices in research on human subjects in Sweden}

\section{Sammanfattning/Abstract}

Mycket av publicerad forskning inom psykologi har inte kunnat reproduceras, vilket bland annat kan bero på tvivelaktiga forskningspraktiker. Öppna forskningspraktiker har föreslagits för att motverka dessa problem. Denna studie syftade till att undersöka prevalensen av uttalade avsikter att använda öppna forskningspraktiker i svensk humanforskning. Vi undersökte förekomsten av uttalad avsikt att använda sig av öppna praktiker i ett stickprov om 102 ansökningar till regionala etikprövningsnämnder i Sverige som godkänts under 2013. Vi undersökte prevalensen av uttalanden om avsikt att (1) publicera resultaten med öppen tillgång, (2) öppet publicera forskningsmaterialet, (3) öppet publicera data och (4) förhandsregistrera studien. 3\% av ansökningar innehöll en uttalad avsikt att publicera under öppen tillgång, och $5 \%$ innehöll referenser till förhandsregistreringar. Uttalanden om avsikt att öppet publicera data och/eller material hittades inte i någon av ansökningarna. En signifikant skillnad mellan typer av öppna praktiker observerades; men inga signifikanta skillnader i parvisa jämförelser mellan praktiker. Låg prevalens skulle bland annat kunna orsakas av att forskare har svaga incitament för att använda öppna praktiker. Framtida studier bör använda nyare etikansökningar och större stickprov.

Nyckelord: etikansökningar, etikprövningsnämnder, forskningskvalitet, öppna forskningspraktiker

Much of published research in psychology has been found irreproducible, possibly caused in part by questionable research practices. Open practices have been suggested as a way to combat these issues. This study aimed to assess the prevalence of declared intent to use open practices in Swedish human subjects research. We investigated the occurrence of declared intent to use open practices in a sample of 102 applications to regional ethical review boards (ERBs) in Sweden approved in 2013. Specifically, we investigated the prevalences of declared intent in applications to (1) publish the report with open access, (2) openly publish research materials, (3) openly publish data, and (4) preregister the study. 3\% of applications stated an intention to publish open reports, and 5\% included references to preregistrations. Statements of intent to publish data and/or materials openly were not found in any applications. A significant difference between types of open practices was identified; however, we observed no statistically significant differences in pairwise comparisons. One cause of low prevalences might be that researchers are disincentivized to use open practices. Future studies should use more recent ERB applications and larger samples.

Keywords: ethics applications, ethics committees, open practices, research integrity 


\title{
Ethical review board-approved protocols and intent to use open practices in research on human subjects in Sweden
}

\author{
Arian Jafari \& Bo Jenner
}

The research community has a tradition of critiquing various aspects of the scientific process with the purpose of improving the quality of scientific results (e.g. de Groot, 2014; Gigerenzer, 2004; Kerr, 1998). Among the more recent concerns is the lack of reproducibility in research. This is often discussed as the replication crisis (Schooler, 2014) or a crisis of confidence (Pashler \& Wagenmakers, 2012), and is a topic of ongoing debate in psychology and other sciences (e.g. Everett \& Earp, 2015; Lindsay, 2015; Nosek, Spies, \& Motyl, 2012; Open Science Collaboration, 2015).

One influential report proposed that the combination of low statistical power and a strong preponderance of positive findings means that most published research findings are false (Ioannidis, 2005). In 2015, an attempt to replicate 100 psychological experiments (Open Science Collaboration, 2015) found that at least $53 \%$ of the original findings could not be reproduced, depending on which metric was used to determine whether a replication was successful. The results were contested by Gilbert, King, Pettigrew and Wilson (2016), citing errors, power issues, and bias. (Their arguments were in turn criticized as flawed and overly optimistic [Anderson et al., 2016].) Feldman Barrett (2015) contended that failure to reproduce does not indicate a crisis, but is merely the scientific process at work; a point also made by the authors of the replication study (Open Science Collaboration, 2015).

Nonetheless, concerns remain. An assessment of effect size distribution and power (Szucs \& Ioannidis, 2017) estimated that $50 \%$ of published findings in psychology and cognitive neuroscience are false positives, because power is generally too low to reliably produce true effects. In fact, underpowered studies are one of the factors that increase the likelihood that findings in a field are false (Button et al., 2013). Other factors include effect sizes within the field, bias and interest, the diversity of investigated relationships, lack of scientific definitions, and how "hot" the field is (Ioannidis, 2005). From this follows that the proportion of incorrect findings will be higher in some fields, in part owing to the characteristics of the subject of study, but also to sample sizes and scientific practices (Ioannidis, 2005).

Although many factors contribute to irreproducibility, the pressure to publish or perish has been identified by several researchers as an important factor (e.g. Everett \& Earp, 2015; Nosek et al., 2012). Researchers rely on grants to do their work, and continued funding often requires successful research in the past, meaning publications and citations. In addition, many who are making a career in academia are equally motivated by prestige and the chance to move ahead. To them, having their work appear in reputable journals and being frequently cited drives the same behavior. The consequence of this is work that is more likely to produce attention-grabbing results, such as effects rather than lack of them, and sensational relationships rather than mundane ones (Smaldino \& McElreath, 2016). This focus also threatens one of the cornerstones of science: direct replication. Repetition of old findings is simply viewed as less exciting than novelty.

Apart from encouraging researchers to test novel hypotheses rather than ones that are potentially more relevant, the pressure to publish may lead to unrigorous science. Questionable research practices among psychology researchers seem to be very prevalent 
(John, Loewenstein, \& Prelec, 2012): 67\% of inquired psychologists in the US admitted they had at times not reported all dependent measures and 58\% had decided whether to collect more data after seeing the results. As many as $43 \%$ reported that they had decided whether to exclude data after considering the impact on the results, and $50 \%$ said they had selectively reported studies. The findings were largely replicated in a recent Italian sample (Agnoli, Wicherts, Veldkamp, Albiero, \& Cubelli, 2017).

These shortcomings potentially have serious consequences outside of the scientific community, as clinical decisions are informed by what is published. If false positive findings are overrepresented in the literature, this may result in the choice of suboptimal or even dangerous treatments. In investigating the prevalence of publication bias in drug-evaluating clinical trials, Suñe, Suñe and Montoro (2013) found that positive results were indeed disseminated at a higher rate than negative results. Positive results were more likely to be published than negative results (85\% compared to 69\%), and were on average published faster (2.1 years compared to 3.2 years). In addition, they found that industry-sponsored studies were less likely to lead to peer-reviewed publications than studies with other sponsors $(12 \%$ and $30 \%$ respectively), suggesting that economic interests lead to negative results being withheld from publication to a higher degree.

\section{Solutions}

Various solutions have been suggested to improve the situation. Begum and Kolstoe (2015) compared applications to a UK National Health Service ethical review board (ERB) with published results, finding that only $32 \%$ of studies had resulted in at least one publication, suggesting considerable publication bias. In comparing published outcomes with outcomes described in ethics applications, they also found inconsistencies in $57 \%$ of the publications. Thus, they suggest that ERBs could play a central role in monitoring publication bias and outcome reporting bias, for instance by having the researchers commit to publishing future results as a condition for approval from the ERB, since ERBs are in a position to act as gatekeepers for research on a national level. They also suggest, as a less time-consuming alternative, that studies comparing ERB applications with published results should be conducted regularly as a way to measure the effectiveness of other bias-reducing approaches in research.

Increasing power by way of larger samples would decrease the risk of spurious effects, and these high-powered studies should primarily attempt to corroborate hypotheses with high probability of being true, so to achieve higher certainty within a field (Ioannidis, 2005). Other recommendations include a priori power calculations, transparent disclosure of methods and analyses, and collaborations (Button et al., 2013).

Open practices. An increasingly common suggestion is to publish reports, data, material, and workflow openly (Munafò, 2016; Nosek \& Bar-Anan, 2012; Nosek et al., 2012). We will refer to the four latter as open practices (OP). Open publication of the final reports, or open report, means that retrieval of the report does not require a journal subscription. The costs associated with publication might instead be covered by authors or their institutions, or by advertising (Nosek \& Bar-Anan, 2012). By open data we refer to the practice of making all data used in analyses publicly available in a digital format, allowing for other researchers to test that the analyses are correct as well as look for other explanations of reported results. Open materials refers to publicly publishing the components required to reproduce the results (e.g. questionnaires, visual aids), while preregistration (i.e. open publishing of workflow) 
refers to the act of registering the study design (including sample size, hypotheses, descriptions of research materials, outcome variables and predictor variables) in a similar publicly available format (Center for Open Science, n.d.).

The impact of preregistration is promising. Kaplan et al. (2015) compared published studies funded by National Heart, Lung, and Blood Institute (NHLBI) before and after 2000, when NHLBI started requiring studies to be registered at clinicaltrials.gov in order to receive funding. Following the introduction of this requirement, significantly positive effects of treatment dropped from $57 \%$ to $8 \%$. Still, the effectiveness of different approaches to increasing transparency of scientific results needs to be further evaluated (Suñé et al., 2013).

In summary, there is a need to improve the way research is conducted in order to increase reproducibility, decrease biases, increase transparency and thus increase the reliability of scientific results. Open practices have been suggested as a way to combat current issues, but, in addition to the effectiveness of these practices being unclear, it is unknown how commonly these practices are applied in research in Sweden.

\section{Aims}

As documented in the preregistration of the current study (Jafari, Jenner, Willén, \& Nilsonne, 2017), the aim was to assess the prevalence of open practices among researchers in Sweden. We analyzed applications to ethical review boards approved during 2013, and investigated to what extent the applicants declared any intent to employ open practices. The following four practices were investigated: open reports, open materials, open data, and preregistration. We hypothesized that the frequency of declared intentions to publish reports under open access would differ from either of the other practices ${ }^{1}$.

The purpose of the study was to explore prevalences of intent to use open practices. To this end, a hypothesis such as prevalences of all the open practices will differ would have been more apt, but because of miscommunication within the research group the preregistration (Jafari et al., 2017) contains the current formulation. This has affected the objective only marginally, and has had no effect on the choice of statistical methods. The exploratory hypothesis above could have been added and evaluated, but this might have complicated decisions about correcting for multiple tests.

\section{Sample}

\section{Method}

Ethical review board-approved applications were gathered from each of the different regional ethical review boards (ERBs) in Sweden. By reviewing ERB applications rather than publications themselves we had access to a sample of research projects in a state where the effect of publication bias had not yet been introduced, and could thus make a better estimation of the use of and attitudes surrounding open practices than by looking at publications only.

Sweden has six regional boards, each consisting of several subcommittees. Our sample consisted of 102 applications, randomly drawn from a larger sample of 360 applications. This larger sample in turn consisted of 20 applications from each subcommittee, and had been randomly selected from a sample frame comprising all applications approved in 2013.

\footnotetext{
${ }^{1}$ The preregistration of this study stated more precisely that the frequency would differ regardless of committee, subcommittee, or study type. The last-mentioned variable was incorrectly described as "whether the study is medical or not", when it actually indicated whether a study was experimental or not. Moreover, these variables were later deemed irrelevant to the research question and were dropped from the analysis.
} 
Applications from Umeå had not been reviewed for confidentiality at the time of coding, and could therefore not be included. The current sample size of 102 applications was based on practical considerations with regard to time available.

\section{Procedure}

Applications were coded by two independent coders. Before commencing coding and analysis of applications, a preregistration of the study was made public on the Open Science Framework (Jafari et al., 2017). Initially, a round of pilot coding was performed on a sample of eight applications, where two coders reviewed the applications independently, in order to highlight potential ambiguities in the coding process before determining the final coding sheet and accompanying codebook. After pilot coding, a variable was added to the coding sheet describing whether the application was searched both manually and with the aid of a text search tool or manually only (Jafari et al., 2017).

During coding, the coders first read the application, searching for declarations of intent to use open practices. Following this, coders attempted to convert scanned applications to searchable text using Google's optical character recognition tool (Google, n.d.). If the conversion was successful, the document was then searched for words from a predefined list of terms (publicera, publish, regist, material, protokoll, protocol) related to open practices.

In coding, only what was apparent from the application-comprising the filled-out template and appendices - was considered; no internet searches were made to gather supplementary information. In addition to a declared intention to make components of the project publicly available, a number of other guidelines were used. These can be found in the updated codebook (Jafari et al., 2017).

\section{Inter-Rater Reliability}

Final statistical analyses were performed on independent coder judgments for variables Study Type and Open Report, while variables Open Materials, Open Data and Preregistration were determined through consensus due to unsatisfactory inter-rater reliability (IRR).

First round of coding. Because the total sample size was initially planned to be 360 applications, and because IRR is commonly determined on the basis of $20 \%$ of the sample, the intention was to code 72 applications. These applications were chosen by creating a list of the total sample, randomly generating 72 integers with random.org and matching them to corresponding indices in the application list. However, time restrictions forced coders to stop earlier than planned, and the first IRR calculations were based on 51 applications.

Calculations. IRR was determined with Cohen's unweighted kappa $(K)$. Various benchmark values exist to decide whether a given kappa is acceptable, such as the ones by Fleiss, Levin, and Paik (2003), where a $K$ above .75 is considered an excellent agreement. Benchmark values are somewhat arbitrary and do not take into account factors that affect the outcome. For example, if rating errors are not random it is possible to achieve high reliability even when accuracy is low. This was considered by Bakeman, McArthur, Quera and Robinson (1997), who suggested kappa levels based on rater accuracy, probability variability, and number of codes. Guided by these recommendations we aimed for an agreement of over .70 for variables Study Type (whether experimental or not), Open Report, Open Materials, Open Data, Preregistration, and an agreement of over .95 for Committee (regional ERB). IRR was not calculated for the following three variables: Registration Number 1, Registration Number 2, and Search Method. These, as well as Study Type and Committee, have no bearing 
on the results; definitions can be found in the codebook (Jafari et al., 2017). The results of the calculation are presented in Table 1.

Conference. After calculating IRR, the coders met and discussed differences in their judgments for all 51 applications. Discrepancies were resolved through consensus. The reason for each discrepancy was identified, and, in the cases where systematic differences were found, stricter definitions for variables and more precise coding procedures were created for future coding. The codebook was then updated (Jafari et al., 2017) to reflect these new guidelines.

Second round of coding. The variables Open Data, Open Materials and Preregistration demonstrated unsatisfactory IRR, and it was therefore decided that an additional 51 applications would be coded. Due to time restrictions, it was decided that these 51 applications would be added to the first 51 and together make up the entire sample (i.e., a total of 102 applications). During this round, the variables with adequate kappa values were coded only by one coder, whereas those with inadequate values were coded by both. Following this, IRR was again calculated and discrepancies reviewed (Jafari et al., 2017). Kappas improved slightly, but were still low for two of the variables: Open Materials and Preregistration (see Table 1). All differences were again solved by consensus. These agreed-upon judgments formed the basis for both variables (Open Materials and Preregistration) with unacceptably low interrater agreement.

Table 1

Interrater Agreements

\begin{tabular}{lcccccc}
\hline & Committee & Study Type & Open Report & Open Materials & Open Data & Preregistration \\
\hline IRR 1 & 1.00 & .71 & .79 & .11 & .46 & .55 \\
IRR 2 & - & - & - & $.54^{1}$ & .78 & $.65^{1}$ \\
\hline
\end{tabular}

Notes. All reported values are Cohen's Unweighted Kappa. ${ }^{1}$ Due to an unsatisfactory level of agreement, this variable was coded in a third round where both coders collaborated to reach a consensus for each application they had previously not agreed on.

\section{Statistical Analysis}

Statistical analyses (Jafari \& Nilsonne, 2017) were conducted with R (R Core Team, 2017). To test the hypothesis that declared intent to publish the report under open access is differently prevalent than the other declared intents, we used Cochran's $Q$ test. This is a nonparametric test used to establish whether the proportions of a dichotomous variable varies between three or more variables that are related. McNemar tests, of which Cochran's $Q$ are an extension, were used to for post hoc testing. At the time for preregistration (Jafari et al., 2017), the plan was to employ Fisher's exact test; however, at the time for conducting the statistical analyses we chose Cochran's $Q$ because the independence of the variables could not be assumed. For example, materials may be more likely to be shared if a repository has already been set up to share data.

Since we were interested in the prevalence of one variable compared to the three others, three tests were required. To address the multiple comparisons problem, this was consequently adjusted for by using false discovery rate correction (Benjamini \& Hochberg, 1995). 


\section{Results}

Out of the reviewed applications, 3/102 (3\%) stated an intention to publish open reports, and 5/102 (5\%) included references to a preregistration. Statements of intent to publish data openly were not found in any of the reviewed applications; the same held true for open materials.

We conducted Cochran's $Q$ test in order to test for differences in frequency between Open Report and the other open practices, which yielded a significant difference indicating that there is a difference between the groups $\left(\chi^{2}(3)=9, p=.029\right)$. When comparing specific pairs of open practices using pairwise McNemar tests, no test yielded significant differences in frequency (as shown in Table 2) after false discovery rate correction for multiple comparisons.

Combined with the descriptive statistics, the results of Cochran's $Q$ test indicated that there was a difference between the frequency of different open practice mentions, and that preregistration mentions occurred more frequently than declarations of intent to publish data or material openly. However, we could not conclude whether there was a difference in frequency between Preregistration and Open Report, nor whether there was a difference between Open Report and Open Data or Open Materials.

Table 2

Comparisons of the Prevalences of the Open Practices

\begin{tabular}{lcc}
\hline & $\chi^{2}$ & $p$ \\
\hline Open Report - Preregistration & .5 & .480 \\
Open Report - Open Data & 3.0 & .125 \\
Open Report - Open Materials & 3.0 & .125 \\
\hline
\end{tabular}

Notes. These are the results of pairwise McNemar tests comparing the frequencies of various open practices, with $p$ values reported after false discovery rate correction.

\section{Discussion}

Applications to ethical review boards offer researchers an opportunity to declare their intent to utilize open practices. The current study assessed the prevalence of such declarations in a sample consisting of applications approved by Swedish ERBs during 2013. More specifically, we investigated the extent to which four open practices were mentioned by the researchers in their ethical applications: preregistration, open data, open material, and open publishing of the final report.

We found that few researchers expressed any intent to employ open practices in their planned research. More specifically, intent to publish data or material was not mentioned even once, while preregistrations and intent to publish under open access were mentioned in five applications and three applications respectively. We found a significant difference between the frequency of mentions of the various types of open practices; however, no significant differences were found between the frequencies of open report and any other group when specific pairs of practices were tested.

It is possible that awareness of the replication crisis, or of other reasons to make science more available, was not as strong four or five years ago when most of the reviewed applications were submitted. Applications approved in 2017 could potentially already be 
indicating greater willingness among researchers to publish openly. On the other hand, a previous study (Tenopir et al., 2011) surveyed researchers (mainly in the United States) about their data sharing practices, and found that as many as $35 \%$ made at least some of their data available on their organization's website, and $15 \%$ said they share most of their data through a national network. If this is any indication, the prevalences in the current study ought to be higher. One possible explanation for this discrepancy is that the decision to adhere to open practices might be one that tends to be made later on in a research project's life cycle, that is at a point where ethics applications have already been submitted and approved.

Although it may seem like open practices would benefit the entire scientific community and beyond, individual researchers might not agree. A review of papers on data-sharing practices (Fecher, Friesike, \& Hebing, 2015) found several explanations for why researchers at times keep data to themselves. Sharing takes time and effort, for example, and may bring with it the need to explain data to the uninitiated who take part of it. Some researchers also fear that others will find errors in their analyses, or that competing institutions will use their hard-earned data to publish before they do. Open publishing of data might also require thinking more carefully about handling data related to research participants. Fears that ERBs will think twice about approving data sharing, warranted or not, could keep researchers from suggesting open publication. By committing to publish data openly, researchers also take on the personal risk of accidentally breaking research confidentiality rules. Making sure one avoids this might require extra work, without presenting any immediate reward for the researchers. Formatting one's data in a way that is usable by other researchers is another timeconsuming task that yields little personal benefit.

Making materials available also requires extra work, but is likely not associated with the same qualms. Together with the fact that materials are already often included as appendices to reports, there are grounds to speculate that they are more commonly published openly than data. The reason that this is not reflected in our results could be that their publication is rarely an ethical consideration and does not need to be brought up with the ERBs.

In 2014, Psychological Science began awarding badges to publications that fulfilled the criteria for open data, open materials and/or preregistration, as a way to offer authors a simple incentive to increase transparency of their research. After badges were introduced, the prevalence of articles using open practices increased significantly. Open data increased from less than 3\% before badges were introduced to $39 \%$ in the first half of 2015 , and open material sharing increased threefold. Before the introduction of badges and the specific criteria required to be awarded them, supposedly openly shared data and materials also ran the risk of being incomplete (Kidwell et al., 2016). These findings indicate that increasing the visibility of open practices and their criteria could be a way of increasing their prevalence within the scientific community.

\section{Coding Discrepancies}

In resolving coding discrepancies, a few cases were notably difficult to agree on. One group of researchers stated that they would make the data available to researchers writing reports. The phrasing could be interpreted as evident intent to provide those interested with the data, and this study did specifically look for stated intent, but there was no explicit mention of open publishing. As the codebook stated that there should exist a mechanism for anyone to access the data without knowing the researchers (Jafari et al., 2017), Open Data was coded as no. In a similar vein, one group of researchers stated they would submit their data to 
Swedish National Data Service. This archiving service works to make data available for future research, but data is not necessarily published openly. Again, because the intent to publish openly was not explicit, Open Data was coded as no. At any rate, this sort of data management is commendable, as most applications simply state that data will be archived locally for a couple of years before being destroyed.

\section{Strengths and Limitations}

In the present study, we attempted to determine the prevalence of intent to use open practices by examining ERB applications. This procedure has the benefit of being unaffected by publication bias, since ethics committee approval is required at a stage in the scientific process before publication bias is introduced.

The final sample size was reduced from the original 360 applications, due to time restrictions. The low prevalence of declared intent to use open practices contributes to uncertainty in the measures. A larger sample might have resulted in more precise estimates.

We found that it was possible to find preregistrations for some of the studies in our sample by performing web searches with certain search terms from the application, such as project names or names of the researchers, even when the application itself gave no indication of the study being preregistered. This suggests that our coding strategy may be overly conservative for this variable. In order to maintain a uniform coding process, we kept a strict routine of only judging the ethics application itself.

Our sample consisted of applications approved by ERBs in 2013. It is possible that the prevalence of declared intent to use open practices has increased in the last few years.

\section{Future Directions}

Because the development of the open science movement and the adoption of the practices explored in the current study deserve continued monitoring, we encourage future studies employing the same method. Preferably, these should make use of a larger sample, not only because prevalences appear to be low, but also because of the wider issue of underpowered studies (Ioannidis, 2005).

Applications to ERBs do not offer comprehensive information about what open practices a research group will use. Future studies trying to determine their prevalences would benefit from including other sources, for example university websites or databases such as clinicaltrials.gov.

A similar future study based on more recent applications might yield different results, in part due to an increased awareness in the scientific community of the potential benefits of open practices in recent years.

\section{Conclusions}

The potential of open practices to increase reproducibility and the overall transparency of the scientific process is promising. The present study shows that Swedish researchers extremely rarely declared any explicit intent in ERB applications to utilize open practices. This may be due to a lack of incentives for individual researchers to pursue open practices. The knowledge and awareness about open practices are however increasing rapidly. We hope and believe that a follow-up study in ten years will show that a majority of researchers explicitly mention intent to employ open practices. Such a change may indeed be crucial to improve the quality of scientific procedures. 


\section{Ethical Considerations}

No individual researchers, research groups or institutions are mentioned in our data. We have not had access to any data that could potentially compromise the integrity of research participants. The regional ERBs used in this study have approved the use of these applications for research purposes. The only exception was the Umeå regional ERB which placed this responsibility with Karolinska Institutet. As these applications had not been cleared for use by a lawyer at the time of this study, they were not included in the sample.

This study has dealt with a topic surrounded by much debate and investigated methods that some find controversial, but it is hard to conceive of a way in which the study in itself might have had any damaging effects in any area.

\section{Acknowledgement}

We would like to express our gratitude to our supervisors Gustav Nilsonne and Rebecca Willén for their invaluable assistance in writing this thesis. Going above and beyond what was required of them in helping us navigate through the many complexities of a new research project, they have provided us with a beautiful humbling experience as well as taught us several valuable lessons in how to deal with unexpected problems with a scientific mindset, and for this we are immensely grateful.

\section{References}

Agnoli, F., Wicherts, J. M., Veldkamp, C. L. S., Albiero, P., \& Cubelli, R. (2017). Questionable research practices among italian research psychologists. PLOS ONE, 12, e0172792. https://doi.org/10.1371/journal.pone.0172792

Anderson, C. J., Bahnik, Š., Barnett-Cowan, M., Bosco, F. A., Chandler, J., Chartier, C. R., ... Zuni, K. (2016). Response to Comment on "Estimating the reproducibility of psychological science." Science, 351, 1037. https://doi.org/10.1126/science.aad9163

Bakeman, R., McArthur, D., Quera, V., \& Robinson, B. F. (1997). Detecting sequential patterns and determining their reliability with fallible observers. Psychological Methods, 2, 357-370. https://doi.org/10.1037/1082-989X.2.4.357

Begum, R., \& Kolstoe, S. (2015). Can UK NHS research ethics committees effectively monitor publication and outcome reporting bias? BMC Medical Ethics, 16, 51. https://doi.org/10.1186/s12910-015-0042-8

Benjamini, Y., \& Hochberg, Y. (1995). Controlling the false discovery rate: a practical and powerful approach to multiple testing. Journal of the Royal Statistical Society. Series B (Methodological), 57, 289-300. Retrieved from http://www.jstor.org.proxy.kib.ki.se/stable/2346101

Button, K. S., Ioannidis, J. P. A., Mokrysz, C., Nosek, B. A., Flint, J., Robinson, E. S. J., \& Munafò, M. R. (2013). Power failure: why small sample size undermines the reliability of neuroscience. Nature Reviews Neuroscience, 14, 365-376. https://doi.org/10.1038/nrn3475

Center for Open Science. (n.d.). Open practice badges details. Retrieved May 17, 2017, from https://cos.io/our-services/open-science-badges-details/

de Groot, A. D. (2014). The meaning of "significance" for different types of research [translated and annotated by Eric-Jan Wagenmakers, Denny Borsboom, Josine Verhagen, Rogier Kievit, Marjan Bakker, Angelique Cramer, Dora Matzke, Don Mellenbergh, and Han L. J. van der Maas]. Acta Psychologica, 148, 188-194. 
https://doi.org/10.1016/j.actpsy.2014.02.001

Everett, J. A. C., \& Earp, B. D. (2015). A tragedy of the (academic) commons: interpreting the replication crisis in psychology as a social dilemma for early-career researchers. Frontiers in Psychology, 6, 1-4. https://doi.org/10.3389/fpsyg.2015.01152

Fecher, B., Friesike, S., \& Hebing, M. (2015). What drives academic data sharing?, 1-25. https://doi.org/10.1371/journal.pone.0118053

Feldman Barrett, L. (2015, September 1). Psychology is not in crisis. New York Times. Retrieved from https://www.nytimes.com/2015/09/01/opinion/psychology-is-not-incrisis.html

Fleiss, J. L., Levin, B., \& Paik, M. C. (2003). The measurement of interrater agreement. In Statistical methods for rates and proportions (3rd ed., pp. 598-626). Hoboken, NJ, USA: John Wiley \& Sons, Inc. https://doi.org/10.1002/0471445428.ch18

Gigerenzer, G. (2004). Mindless statistics. Journal of Socio-Economics, 33, 587-606. https://doi.org/10.1016/j.socec.2004.09.033

Gilbert, D. T., King, G., Pettigrew, S., \& Wilson, T. D. (2016). Comment on "Estimating the reproducibility of psychological science." Science, 351, 1037-b.

https://doi.org/10.1126/science.aad7243

Google. (n.d.). Convert PDF and photo files to text. Retrieved May 17, 2017, from https://support.google.com/drive/answer/176692?co=GENIE.Platform\%3DDesktop\&hl= en $\% 0 \mathrm{D}$

Ioannidis, J. P. A. (2005). Why most published research findings are false. PLOS Medicine, 2, e124. https://doi.org/10.1371/journal.pmed.0020124

Jafari, A., Jenner, B., Willén, R. M., \& Nilsonne, G. (2017). Ethical review board-approved protocols and intent to use open practices in research on human subjects in Sweden. Retrieved from osf.io/qxbsz

Jafari, A., \& Nilsonne, G. (2017). Xanindorf/intent-open-practices v1.0 [Data set]. Zenodo. https://doi.org/10.5281/zenodo.574209

John, L. K., Loewenstein, G., \& Prelec, D. (2012). Measuring the prevalence of questionable research practices with incentives for truth telling. Psychological Science, 23, 524-532. https://doi.org/10.1177/0956797611430953

Kaplan, R. M., Irvin, V. L., Waters, T., Pettinger, M., Rossouw, J., \& Anderson, G. (2015). Likelihood of null effects of large NHLBI clinical trials has increased over time. PLOS ONE, 10, e0132382. https://doi.org/10.1371/journal.pone.0132382

Kerr, N. L. (1998). HARKing: Hypothesizing After the Results are Known. Personality and Social Psychology Review, 2, 196-217. https://doi.org/10.1207/s15327957pspr0203_4

Kidwell, M. C., Lazarević, L. B., Baranski, E., Hardwicke, T. E., Piechowski, S., Falkenberg, L.-S., ... Nosek, B. A. (2016). Badges to acknowledge open practices: a simple, lowcost, effective method for increasing transparency. PLOS Biology, 14, e1002456. https://doi.org/10.1371/journal.pbio.1002456

Lindsay, S. (2015). Replication in psychological science. Psychological Science, 26, 18271832. https://doi.org/10.1177/0956797615616374

Munafò, M. (2016). Open science and research reproducibility. Ecancermedicalscience, 10, ed56. https://doi.org/10.3332/ecancer.2016.ed56

Nosek, B. A., \& Bar-Anan, Y. (2012). Scientific Utopia: I. Opening scientific communication. Psychological Inquiry, 23, 217-243. https://doi.org/10.1080/1047840X.2012.692215

Nosek, B. A., Spies, J. R., \& Motyl, M. (2012). Scientific Utopia: II. Restructuring incentives 
and practices to promote truth over publishability. Perspectives on Psychological Science, 7, 615-631. https://doi.org/10.1177/1745691612459058

Open Science Collaboration. (2015). Estimating the reproducibility of psychological science. Science, 349, aac4716-aac4716. https://doi.org/10.1126/science.aac4716

Pashler, H., \& Wagenmakers, E. J. (2012). Editors' introduction to the special section on replicability in psychological science: A crisis of confidence? Perspectives on Psychological Science, 7, 528-530. https://doi.org/10.1177/1745691612465253

R Core Team. (2017). R: A language and environment for statistical computing. Vienna, Austria: R Foundation for Statistical Computing. Retrieved from http://www.rproject.org/

Schooler, J. (2014). Metascience could rescue the "replication crisis." Nature, 515, 9. https://doi.org/doi: 10.1038/515009a

Smaldino, P. E., \& McElreath, R. (2016). The natural selection of bad science. Royal Society Open Science, 3, 160384. https://doi.org/10.1098/rsos.160384

Suñé, P., Suñé, J. M., \& Montoro, J. B. (2013). Positive outcomes Influence the rate and time to publication, but not the impact factor of publications of clinical trial results. PLOS $O N E, 8$, e54583. https://doi.org/10.1371/journal.pone.0054583

Szucs, D., \& Ioannidis, J. P. A. (2017). Empirical assessment of published effect sizes and power in the recent cognitive neuroscience and psychology literature. PLOS Biology, 15, e2000797. https://doi.org/10.1371/journal.pbio.2000797

Tenopir, C., Allard, S., Douglass, K., Aydinoglu, A. U., Wu, L., Read, E., ... Frame, M. (2011). Data sharing by scientists: practices and perceptions, 6, 1-21.

https://doi.org/10.1371/journal.pone.0021101 